American Journal of Animal and Veterinary Sciences 5 (4): 237-243, 2010

ISSN 1557-4555

(C) 2010 Science Publications

\title{
Effects of Dietary Inclusion of Guar Meal Supplemented by ß-Mannanase on Performance of Laying Hens, Egg Quality Characteristics and Diacritical Counts of White Blood Cells
}

\author{
Mohammad Ehsani and Mehran Torki \\ Department of Animal Science, Faculty of Agriculture, Razi University, \\ Imam Avenue, Postal Code: 6715685418, Kermanshah, Iran
}

\begin{abstract}
Problem statement: Using Guar Meal (GM) in poultry diets has being limited because of having $\beta$-mannan, one of the Nonstarch Polysaccharides (NSP). In this study we try evaluating effects of enzyme supplementation of GM-included diets on productive performance of laying hens. Approach: A total number of 144 Lohmann LSL-Lite hens were divided in 24 cages $(n=6)$. Based on a $3 \times 2$ factorial arrangement of treatments, six iso-caloric and iso-nitrogenous diets including 3 levels of $\operatorname{GM}\left(0.0,35.0\right.$ and $\left.70.0 \mathrm{~g} \mathrm{~kg}^{-1}\right)$ with and without enzyme (Hemicell ${ }^{\circledR}$ a $\beta$-mannanase-based enzyme, 0.0 and $0.6 \mathrm{~g} \mathrm{~kg}^{-1}$ ) were assigned to hens in 4 cages (replicates). Data was analyzed based on completely randomized design using GLM procedure of SAS. Results: Dietary GM inclusion significantly affected on Egg Production (EP) on weeks 2, 4 and 6 as well as the overall trail period. Hens fed the GM-included diets did have decreased EP compared to hens fed the control diet. Almost the same trend was observed in terms of Egg Mass (EM); so that hens fed the GM-included diets showed decreased EM compared to the hens fed the control diet. Enzyme supplementation did not have significant effect on EP in the present experiment, but EM was significantly improved in the hens fed the $\beta$-mannanase-supplemented diets on weeks 3, 6 and the overall experimental period. Dietary inclusion GM increased Feed Conversion Ratio (FCR) of laying hens compared to the hens fed the control diets on weeks 2, 4, 6 and overall trial period. Conclusion/Recommendations: Including GM in laying hens' diets more than $3 \%$ may decrease productive performance. Supplementing cornsoybean or corn-soybean-GM diets by $\beta$-mannanase would have beneficial effects on performance of hens especially in terms of FCR and EP.
\end{abstract}

Key words: Guar meal, enzyme, $\beta$-mannanase, performance, egg characteristics, laying hens

\section{INTRODUCTION}

Guar Meal (GM), a relatively inexpensive high protein meal, produced as a by-product of guar gum manufacture. The protein content of GM ranges between $33-60 \%$ depending on fraction type. GM results from combinations of two fractions, the germ and hull. The germ and hull constitute approximately 44 and $21 \%$ of the guar bean, respectively. However, the germ and hull proportions of the guar bean are not consistent with the relative amounts of the fractions mixed in guar meal. Also, the degree of contamination of the germ and hull fractions with guar gum is not equivalent within these proportions when mixed into commercial GM. Guar gum residue contained in the meal increases the viscosity of digesta, thereby decreasing growth and feed efficiency.
Improving poultry performance by dietary manipulation has been the goal of nutritionists. Modification NRC nutrient recommendations (Maroufyan et al., 2010; Fanooci and Torki, 2010), using feed additives like enzymes (Zangiabadi and Torki, 2010), organic acids (Mahdavi and Torki, 2009) or medicinal plants (Ghasemi et al., 2010; Najafi and Torki, 2010) has been reported by other researchers. A patented enzyme product ( $\beta$-mannanase, Hemicell) has been shown to improve feed conversion in cornsoybean diets fed to layers (Zangiabadi and Torki, 2009). The mechanism of $\beta$-mannanase is to degrade $\beta$ mannan, which is an antinutritional factor existing in many legumes, including soybean and canola meals. Dietary inclusion of Hemicell has improved broiler performance of broilers fed corn-soybean meal- or corn-soybean meal-palm date-included diets (Zangiabadi and Torki, 2010).

Corresponding Author: Mehran Torki, Department of Animal Science, Faculty of Agriculture, Razi University, Imam Avenue, Postal Code: 6715685418, Kermanshah, Iran 
The objectives of the present study were to investigate effects of dietary including graded levels of GM supplemented by enzyme on productive performance of laying hens, egg quality characteristics and diacritical counts of white blood cells.

\section{MATERIALS AND METHODS}

A total number of 144 Lohmann LSL-Lite hens were divided in 24 cages $(n=6)$ with almost equal distribution of average body weight and egg production among cages. Hens in 4 cages (replicates) were assigned to feed on one the 6 experimental diets. Based on a $3 \times 2$ factorial arrangement of treatments, 6 isocaloric and iso-nitrogenous diets $\left(\mathrm{ME}=2720 \mathrm{Kcal} \mathrm{kg}^{-1}\right.$ and $\left.\mathrm{CP}=145 \mathrm{~g} \mathrm{~kg}^{-1}\right)$ including $\mathrm{GM}(0.0,35.0$ and 70.0 $\left.\mathrm{g} \mathrm{kg} \mathrm{kg}^{-1}\right)$ and enzyme $\left(0.0\right.$ and $\left.0.6 \mathrm{~g} \mathrm{~kg}^{-1}\right)$ were formulated (Table 1). Collected data of Feed Intake (FI), Egg Production (EP), Egg Mass (EM) and calculated Feed Conversion Ratio (FCR) during 6 week trial period was analyzed based on completely randomized design using GLM procedure of SAS.

\section{RESULTS}

Effects of dietary GM inclusion and enzyme supplementation on EP, FI, FCR, Egg Weight (EW) and EM during experimental period (6 weeks) are presented in Table 1-6, respectively. There was no interaction between dietary GM inclusion and enzyme supplementation on any of productive performance and egg quality traits $(\mathrm{p}>0.05)$. Dietary GM inclusion significantly affected on EP on weeks 2, 4 and 6 as well as the overall trail period (weeks 1-6). Hens fed the GM-included diets did have decreased EP compared to hens fed the control diet. Almost the same trend was observed in terms of EM; so that hens fed the GMincluded diets showed decreased EM compared to hens fed the control diet (Table 6).

Table 1: Composition of the experimental diets

\begin{tabular}{|c|c|c|c|c|c|c|}
\hline \multirow{3}{*}{$\begin{array}{l}\text { Guar meal (g/100 g diet) } \\
\text {--omicel (g/100 g diet) }\end{array}$} & \multicolumn{6}{|c|}{$\mathrm{g} / 100 \mathrm{~g}$ diet } \\
\hline & \multicolumn{2}{|l|}{0.0} & \multicolumn{2}{|l|}{3.5} & \multicolumn{2}{|l|}{7.0} \\
\hline & 0.00 & 0.06 & 0.00 & 0.06 & 0.00 & 0.06 \\
\hline \multicolumn{7}{|l|}{ Feed ingredients } \\
\hline Corn & 61.37 & 61.37 & 60.60 & 60.60 & 59.81 & 59.81 \\
\hline Soybean meal & 13.15 & 13.14 & 8.61 & 8.61 & 3.99 & 3.99 \\
\hline Date pits & 10.00 & 10.00 & 10.00 & 10.00 & 10.00 & 10.00 \\
\hline Fish meal & 4.42 & 4.42 & 4.42 & 4.42 & 4.42 & 4.42 \\
\hline Soybean oil & 0.68 & 0.68 & 0.68 & 0.68 & 0.68 & 0.68 \\
\hline Dicalcium phosphate & 1.08 & 1.08 & 1.10 & 1.10 & 1.11 & 1.11 \\
\hline Limestone & 8.45 & 8.45 & 8.46 & 8.46 & 8.46 & 8.46 \\
\hline Common salt & 0.23 & 0.23 & 0.23 & 0.23 & 0.24 & 0.24 \\
\hline Guar meal & 0.00 & 0.00 & 3.50 & 3.50 & 7.00 & 7.00 \\
\hline Hemicell & 0.00 & 0.06 & 0.00 & 0.06 & 0.00 & 0.06 \\
\hline Vit. and Min. Premix ${ }^{1}$ & 0.25 & 0.25 & 0.25 & 0.25 & 0.25 & 0.25 \\
\hline Sand & 0.05 & - & 1.91 & 1.85 & 3.79 & 3.73 \\
\hline DL-Methionine & 0.06 & 0.07 & - & - & - & - \\
\hline \multicolumn{7}{|l|}{ Calculated analyses } \\
\hline $\mathrm{ME}(\mathrm{Kcal} / \mathrm{kg})$ & 2720.00 & 2720.00 & 2720.00 & 2720.00 & 2720.00 & 2720.00 \\
\hline Crude protein (\%) & 14.58 & 14.58 & 14.58 & 14.58 & 14.58 & 14.58 \\
\hline Ether extract (\%) & 3.98 & 3.98 & 4.11 & 4.11 & 4.23 & 4.23 \\
\hline Crude fiber (\%) & 7.13 & 7.13 & 6.96 & 6.96 & 6.77 & 6.77 \\
\hline Calcium (\%) & 3.75 & 3.75 & 3.75 & 3.75 & 3.75 & 3.75 \\
\hline Available P (\%) & 0.29 & 0.29 & 0.29 & 0.29 & 0.29 & 0.29 \\
\hline Lys (\%) & 0.74 & 0.74 & 0.69 & 0.69 & 0.65 & 0.65 \\
\hline Met (\%) & 0.33 & 0.33 & 0.30 & 0.30 & 0.33 & 0.33 \\
\hline Met and Cys (\%) & 0.56 & 0.56 & 0.59 & 0.59 & 0.67 & 0.67 \\
\hline
\end{tabular}


American J. Animal \& Vet. Sci., 5 (4): 237-243, 2010

Table 2: Effects of dietary guar meal inclusion $\left(0,35.0\right.$ and $\left.70 \mathrm{~g} \mathrm{~kg}^{-1}\right)$ and enzyme supplementation $\left(0.0\right.$ and $\left.0.6 \mathrm{~g} \mathrm{~kg}^{-1}\right)$ on egg production $(\%)$

\begin{tabular}{|c|c|c|c|c|c|c|c|c|}
\hline \multirow{2}{*}{ Weeks of trial } & & \multicolumn{7}{|c|}{ Egg production (\%) } \\
\hline & & Week 1 & Week 2 & Week 3 & Week 4 & Week 5 & Week 6 & Weeks 1-6 \\
\hline \multicolumn{9}{|l|}{ Treatment } \\
\hline \multicolumn{9}{|l|}{ Enzyme } \\
\hline 0.00 & & 77.770 & 63.880 & 67.260 & 67.260 & 68.650 & 67.260 & 68.680 \\
\hline 0.06 & & 79.760 & 68.250 & 73.610 & 70.630 & 75.190 & 74.600 & 73.670 \\
\hline \multicolumn{9}{|l|}{ Guar meal (\%) } \\
\hline 0.00 & & 80.950 & $75.590 \mathrm{a}$ & 76.190 & $75.890 \mathrm{a}$ & 76.780 & $75.590 \mathrm{a}$ & $76.830 \mathrm{a}$ \\
\hline 3.50 & & 77.670 & $63.390 \mathrm{~b}$ & 70.230 & $66.660 \mathrm{ab}$ & 68.150 & $71.420 \mathrm{ab}$ & $69.590 \mathrm{~b}$ \\
\hline 7.00 & & 77.670 & $59.220 \mathrm{~b}$ & 64.880 & $64.280 \mathrm{~b}$ & 70.830 & $65.770 \mathrm{~b}$ & $69.590 \mathrm{~b}$ \\
\hline Guar meal (\%) & Enzyme & & & & & & & \\
\hline 0.00 & 0.00 & 79.760 & 75.000 & 74.400 & 76.780 & 75.000 & 74.400 & 75.890 \\
\hline 0.00 & 0.06 & 82.140 & 76.190 & 77.970 & 75.000 & 78.570 & 76.780 & 77.780 \\
\hline 3.50 & 0.00 & 74.400 & 60.110 & 66.070 & 61.900 & 63.690 & 64.880 & 65.170 \\
\hline 3.50 & 0.06 & 80.950 & 66.660 & 74.400 & 71.420 & 72.620 & 77.970 & 74.000 \\
\hline 7.00 & 0.00 & 79.160 & 56.550 & 61.310 & 63.090 & 67.260 & 62.500 & 64.980 \\
\hline 7.00 & 0.06 & 76.190 & 61.900 & 68.450 & 65.470 & 74.400 & 69.050 & 69.240 \\
\hline SEM MSEM & & 6.434 & 7.664 & 10.047 & 9.148 & 9.723 & 7.182 & 6.009 \\
\hline \multicolumn{2}{|l|}{ Source of variation } & \multicolumn{7}{|l|}{ p-values } \\
\hline Guar meal & & 0.514 & 0.001 & 0.107 & 0.048 & 0.219 & 0.042 & 0.012 \\
\hline Enzyme & & 0.460 & 0.179 & 0.139 & 0.378 & 0.116 & 0.022 & 0.056 \\
\hline Enzyme $\times$ Guar meal & 0.354 & 0.766 & 0.886 & 0.472 & 0.855 & 0.344 & 0.514 & \\
\hline
\end{tabular}

a-b: Means within a column (within main effects) with no common superscript differ significantly $(\mathrm{p}<0.05), \mathrm{SEM}=$ Standard Error of Means

Table 3: Effects of dietary guar meal inclusion $\left(0,35.0\right.$ and $\left.70 \mathrm{~g} \mathrm{~kg}^{-1}\right)$ and enzyme supplementation $\left(0.0\right.$ and $\left.0.6 \mathrm{~g} \mathrm{~kg}^{-1}\right)$ on feed intake

\begin{tabular}{|c|c|c|c|c|c|c|c|c|}
\hline \multirow[b]{2}{*}{ Weeks of trial } & & \multicolumn{7}{|c|}{ Feed intake $\left(\mathrm{g} \mathrm{hen}^{-1}\right.$ day $\left.^{-1}\right)$} \\
\hline & & Week 1 & Week 2 & Week 3 & Week 4 & Week 5 & Week 6 & Weeks 1-6 \\
\hline \multicolumn{9}{|l|}{ Treatment } \\
\hline \multicolumn{9}{|l|}{ Enzyme } \\
\hline 0.00 & & $119.470 \mathrm{a}$ & 119.470 & 119.730 & 119.890 & 119.200 & 119.780 & 119.590 \\
\hline 0.06 & & $119.040 \mathrm{~b}$ & 118.730 & 119.400 & 119.360 & 118.930 & 119.180 & 119.110 \\
\hline \multicolumn{9}{|l|}{ Guar meal } \\
\hline 0.00 & & 119.410 & 119.770 & 119.830 & 119.370 & 119.240 & 119.280 & 119.480 \\
\hline 3.50 & & 119.220 & 119.130 & 119.770 & 119.920 & 119.270 & 119.490 & 119.470 \\
\hline 7.00 & & 119.120 & 118.400 & 119.090 & 119.580 & 118.700 & 119.670 & 119.090 \\
\hline Guar meal & Enzyme & & & & & & & \\
\hline 0.00 & 0.00 & 119.700 & 119.820 & 119.880 & 120.000 & 120.000 & 120.000 & 119.900 \\
\hline 0.00 & 0.06 & 119.130 & 119.730 & 119.790 & 118.750 & 118.480 & 118.570 & 119.070 \\
\hline 3.50 & 0.00 & 119.400 & 119.370 & 120.000 & 119.850 & 119.730 & 120.000 & 119.720 \\
\hline 3.50 & 0.06 & 119.050 & 118.900 & 119.550 & 119.850 & 118.810 & 118.980 & 119.210 \\
\hline 7.00 & 0.00 & 119.310 & 119.220 & 119.310 & 119.820 & 117.880 & 119.340 & 119.150 \\
\hline 7.00 & 0.06 & 118.930 & 117.580 & 118.870 & 119.340 & 119.520 & 120.000 & 119.040 \\
\hline SEM & & 0.387 & 1.327 & 1.077 & 0.826 & 2.158 & 1.525 & 0.755 \\
\hline Source of variation & & p-values & & & & & & \\
\hline Guar meal & & 0.329 & 0.147 & 0.331 & 0.423 & 0.842 & 0.880 & 0.518 \\
\hline Enzyme & & 0.013 & 0.192 & 0.467 & 0.136 & 0.765 & 0.352 & 0.136 \\
\hline Enzyme $\times$ Guar meal & & 0.847 & 0.492 & 0.929 & 0.263 & 0.322 & 0.372 & 0.646 \\
\hline
\end{tabular}

a-b: Means within a column (within main effects) with no common superscript differ significantly $(\mathrm{p}<0.05), \mathrm{SEM}=$ Standard Error of Means

Although, enzyme supplementation did not have significant effect on EP in the present experiment (Table 2), EM was significantly improved in hens fed the $\beta$-mannanase-supplemented diets on weeks 3, 6 and the overall experimental period (weeks 1-6, Table 6). Hens fed the GM-included diets had decreased FI compared to hens fed the control diet on week 1 of the trial; however, FI of hens during week 2-6 did not significantly affected by dietary GM inclusion or enzyme supplementation (Table 3 ). As it is presented in Table 4, including GM to diets increased FCR of laying hens compared to hens fed the control diets on weeks 2, 4,6 and overall trial period (weeks 1-6). In the present study, dietary supplementation by $\beta$-mannanase improved FCR on weeks 3, 6 and the overall experimental period. The results of dietary treatment on the measured egg quality characteristics were shown in Table 6. 
American J. Animal \& Vet. Sci., 5 (4): 237-243, 2010

Table 4: Effects of dietary guar meal inclusion $\left(0,35.0\right.$ and $\left.70 \mathrm{~g} \mathrm{~kg}^{-1}\right)$ and enzyme supplementation $\left(0.0\right.$ and $\left.0.6 \mathrm{~g} \mathrm{~kg}^{-1}\right)$ on Feed Conversion Ratio (FCR)

\begin{tabular}{|c|c|c|c|c|c|c|c|c|}
\hline \multirow[b]{2}{*}{ Weeks of trial } & & \multicolumn{7}{|c|}{ Feed conversion ratio ( $\mathrm{g}$ feed: $\mathrm{g}$ egg) } \\
\hline & & Week 1 & Week 2 & Week 3 & Week 4 & Week 5 & Week 6 & Weeks 1-6 \\
\hline \multicolumn{9}{|l|}{ Treatment } \\
\hline \multicolumn{9}{|l|}{ Enzyme } \\
\hline 0.00 & & 2.430 & 3.08 & $2.960 \mathrm{a}$ & 2.940 & 2.810 & $2.940 \mathrm{a}$ & $2.860 \mathrm{a}$ \\
\hline 0.06 & & 2.370 & 2.83 & $2.590 \mathrm{~b}$ & 2.760 & 2.560 & $2.560 \mathrm{~b}$ & $2.610 \mathrm{~b}$ \\
\hline \multicolumn{9}{|l|}{ Guar meal } \\
\hline 0.00 & & 2.340 & $2.53 b$ & 2.520 & $2.500 \mathrm{~b}$ & 2.460 & $2.540 \mathrm{~b}$ & $2.480 \mathrm{~b}$ \\
\hline 3.50 & & 2.380 & $3.03 \mathrm{a}$ & 2.760 & $2.910 \mathrm{ab}$ & 2.840 & $2.730 \mathrm{ab}$ & $2.770 \mathrm{a}$ \\
\hline 7.00 & & 2.470 & $3.31 \mathrm{a}$ & 3.040 & $3.130 \mathrm{a}$ & 2.740 & $2.980 \mathrm{a}$ & $2.950 \mathrm{a}$ \\
\hline Guar meal & Enzyme & & & & & & & \\
\hline 0.00 & 0.00 & 2.420 & 2.58 & 2.650 & 2.470 & 2.540 & 2.650 & 2.550 \\
\hline 0.00 & 0.06 & 2.270 & 2.48 & 2.390 & 2.530 & 2.390 & 2.440 & 2.420 \\
\hline 3.50 & 0.00 & 2.490 & 3.19 & 2.930 & 3.100 & 3.010 & 2.990 & 2.950 \\
\hline 3.50 & 0.06 & 2.280 & 2.87 & 2.590 & 2.720 & 2.680 & 2.470 & 2.600 \\
\hline 7.00 & 0.00 & 2.390 & 3.48 & 3.290 & 3.240 & 2.880 & 3.170 & 3.080 \\
\hline 7.00 & 0.06 & 2.560 & 3.14 & 2.790 & 3.020 & 2.600 & 2.780 & 2.820 \\
\hline SEM & & 0.192 & 0.393 & 0.404 & 0.445 & 0.356 & 0.314 & 0.249 \\
\hline Source of variation & & p-values & & & & & & \\
\hline Guar meal & & 0.408 & 0.003 & 0.058 & 0.034 & 0.115 & 0.041 & 0.005 \\
\hline Enzyme & & 0.425 & 0.134 & 0.039 & 0.339 & 0.099 & 0.009 & 0.025 \\
\hline Enzyme $\times$ Guar meal & & 0.133 & 0.800 & 0.832 & 0.609 & 0.875 & 0.621 & 0.692 \\
\hline
\end{tabular}

Table 5: Effects of dietary guar meal inclusion $\left(0,35.0\right.$ and $\left.70 \mathrm{~g} \mathrm{~kg}^{-1}\right)$ and enzyme supplementation $\left(0.0\right.$ and $\left.0.6 \mathrm{~g} \mathrm{~kg}^{-1}\right)$ on average egg weight

\begin{tabular}{|c|c|c|c|c|c|c|c|c|}
\hline \multirow[b]{2}{*}{ Weeks of trial } & & \multicolumn{7}{|c|}{ Egg weight (g) } \\
\hline & & Week 1 & Week 2 & Week 3 & Week 4 & Week 5 & Week 6 & Weeks 1-6 \\
\hline \multicolumn{9}{|l|}{ Treatment } \\
\hline \multicolumn{9}{|l|}{ Enzyme } \\
\hline 0.00 & & 63.55 & 62.40 & 62.260 & 62.747 & 62.910 & 61.760 & 62.600 \\
\hline 0.06 & & 63.36 & 62.48 & 63.300 & 62.152 & 62.810 & 63.540 & 62.940 \\
\hline \multicolumn{9}{|l|}{ Guar meal } \\
\hline 0.00 & & 63.60 & 62.72 & 63.600 & 63.070 & 63.720 & 62.450 & 63.190 \\
\hline 3.50 & & 64.32 & 62.94 & 62.710 & 62.930 & 62.750 & 62.640 & 63.050 \\
\hline 7.00 & & 62.46 & 61.67 & 62.030 & 61.330 & 62.100 & 62.860 & 62.070 \\
\hline Guar meal & Enzyme & & & & & & & \\
\hline 0.00 & 0.00 & $62.68 \mathrm{ab}$ & 62.01 & 62.400 & 63.200 & 63.400 & 61.070 & 62.460 \\
\hline 0.00 & 0.06 & $64.52 \mathrm{a}$ & 63.43 & 64.800 & 62.940 & 64.040 & 63.830 & 63.930 \\
\hline 3.50 & 0.00 & $64.22 \mathrm{a}$ & 63.26 & 63.020 & 63.450 & 63.220 & 62.980 & 63.360 \\
\hline 3.50 & 0.06 & $64.42 \mathrm{a}$ & 62.62 & 62.400 & 62.420 & 62.280 & 62.300 & 62.740 \\
\hline 7.00 & 0.00 & $63.76 a$ & 61.94 & 61.350 & 61.580 & 62.090 & 61.220 & 61.990 \\
\hline 7.00 & 0.06 & $61.16 b$ & 61.41 & 62.720 & 61.090 & 62.100 & 64.500 & 62.160 \\
\hline SEM & & 1.920 & 2.131 & 2.712 & 2.612 & 2.278 & 3.485 & 2.137 \\
\hline Source of variation & & $P$ values & & & & & & \\
\hline Guar meal & & 0.095 & 0.462 & 0.525 & 0.356 & 0.378 & 0.972 & 0.534 \\
\hline Enzyme & & 0.780 & 0.926 & 0.357 & 0.583 & 0.914 & 0.225 & 0.703 \\
\hline Enzyme $\times$ Guar meal & & 0.040 & 0.564 & 0.537 & 0.954 & 0.787 & 0.481 & 0.622 \\
\hline
\end{tabular}

a-b: Means within a column (within main effects) with no common superscript differ significantly ( $<0.05$ ), SEM = Standard Error of Means

As it is shown in Table 7, among the egg quality traits, only the thickness of egg shell was significantly affected by dietary GM inclusion. Hens fed diet included 7\% GM did have lower egg shell thickness compared to hens fed the other two experimental diets.
Enzyme supplementation did not have any beneficial effects on the measured egg traits in this study.

The results of dietary effects on the white blood cell count in the present trial are shown in Table 8. There was no significant effect of dietary treatment on the white blood cell count. 
American J. Animal \& Vet. Sci., 5 (4): 237-243, 2010

Table 6: Effects of dietary guar meal inclusion $\left(0,35.0\right.$ and $\left.70 \mathrm{~g} \mathrm{~kg}^{-1}\right)$ and enzyme supplementation $\left(0.0\right.$ and $\left.0.6 \mathrm{~g} \mathrm{~kg}^{-1}\right)$ on egg mass

\begin{tabular}{|c|c|c|c|c|c|c|c|c|}
\hline \multirow[b]{2}{*}{ Weeks of trial } & & \multicolumn{7}{|c|}{ Egg mass $\left(\mathrm{g} \mathrm{hen}^{-1}\right.$ day $\left.^{-1}\right)$} \\
\hline & & Week 1 & Week 2 & Week 3 & Week 4 & Week 5 & Week 6 & Weeks 1-6 \\
\hline \multicolumn{9}{|l|}{ Treatment } \\
\hline \multicolumn{9}{|l|}{ Enzyme } \\
\hline 0.00 & & 49.360 & 39.750 & $41.640 \mathrm{~b}$ & 42.100 & 43.150 & $41.420 \mathrm{~b}$ & $42.910 \mathrm{~b}$ \\
\hline 0.06 & & 50.600 & 42.720 & $46.430 \mathrm{a}$ & 43.780 & 47.060 & $46.970 \mathrm{a}$ & $46.260 \mathrm{a}$ \\
\hline \multicolumn{9}{|l|}{ Guar meal } \\
\hline 0.00 & & 51.450 & $47.390 \mathrm{a}$ & $48.220 \mathrm{a}$ & $47.790 \mathrm{a}$ & 48.760 & 47.160 & $48.460 \mathrm{a}$ \\
\hline 3.50 & & 50.250 & $39.980 \mathrm{~b}$ & $43.910 \mathrm{ab}$ & $41.740 \mathrm{~b}$ & 42.760 & 44.640 & $43.880 \mathrm{~b}$ \\
\hline 7.00 & & 48.240 & $36.330 \mathrm{~b}$ & $39.980 \mathrm{~b}$ & $39.280 \mathrm{~b}$ & 43.790 & 40.800 & $41.400 \mathrm{~b}$ \\
\hline Guar meal & Enzyme & & & & & & & \\
\hline 0.00 & 0.00 & 50.000 & 46.430 & 46.190 & 48.570 & 47.560 & 45.390 & 47.350 \\
\hline 0.00 & 0.06 & 52.910 & 48.360 & 50.260 & 47.020 & 49.970 & 48.930 & 49.570 \\
\hline 3.50 & 0.00 & 48.120 & 38.060 & 41.420 & 39.190 & 40.290 & 40.830 & 41.320 \\
\hline 3.50 & 0.06 & 52.380 & 41.900 & 46.390 & 44.280 & 45.230 & 48.450 & 46.440 \\
\hline 7.00 & 0.00 & 49.970 & 34.760 & 37.320 & 38.540 & 41.600 & 38.060 & 40.045 \\
\hline 7.00 & 0.06 & 46.510 & 37.910 & 42.650 & 40.030 & 45.980 & 43.540 & 42.770 \\
\hline SEM & & 4.143 & 4.879 & 5.287 & 5.282 & 5.590 & 4.860 & 3.253 \\
\hline Source of variation & & p-values & & & & & & \\
\hline Guar meal & & 0.316 & 0.000 & 0.020 & 0.013 & 0.099 & 0.053 & 0.001 \\
\hline Enzyme & & 0.473 & 0.152 & 0.039 & 0.447 & 0.104 & 0.012 & 0.021 \\
\hline Enzyme $\times$ Guar meal & & 0.167 & 0.924 & 0.970 & 0.468 & 0.893 & 0.707 & 0.643 \\
\hline
\end{tabular}

a-b: Means within a column (within main effects) with no common superscript differ significantly ( $<<0.05$ ), SEM = Standard Error of Means

Table 7: Effects of dietary guar meal inclusion $\left(0,35.0\right.$ and $\left.70 \mathrm{~g} \mathrm{~kg}^{-1}\right)$ and enzyme supplementation $\left(0.0\right.$ and $\left.0.6 \mathrm{~g} \mathrm{~kg}^{-1}\right)$ on egg quality characteristics (egg index, yolk index, Haugh unit, egg shell weight and egg shell thickness)

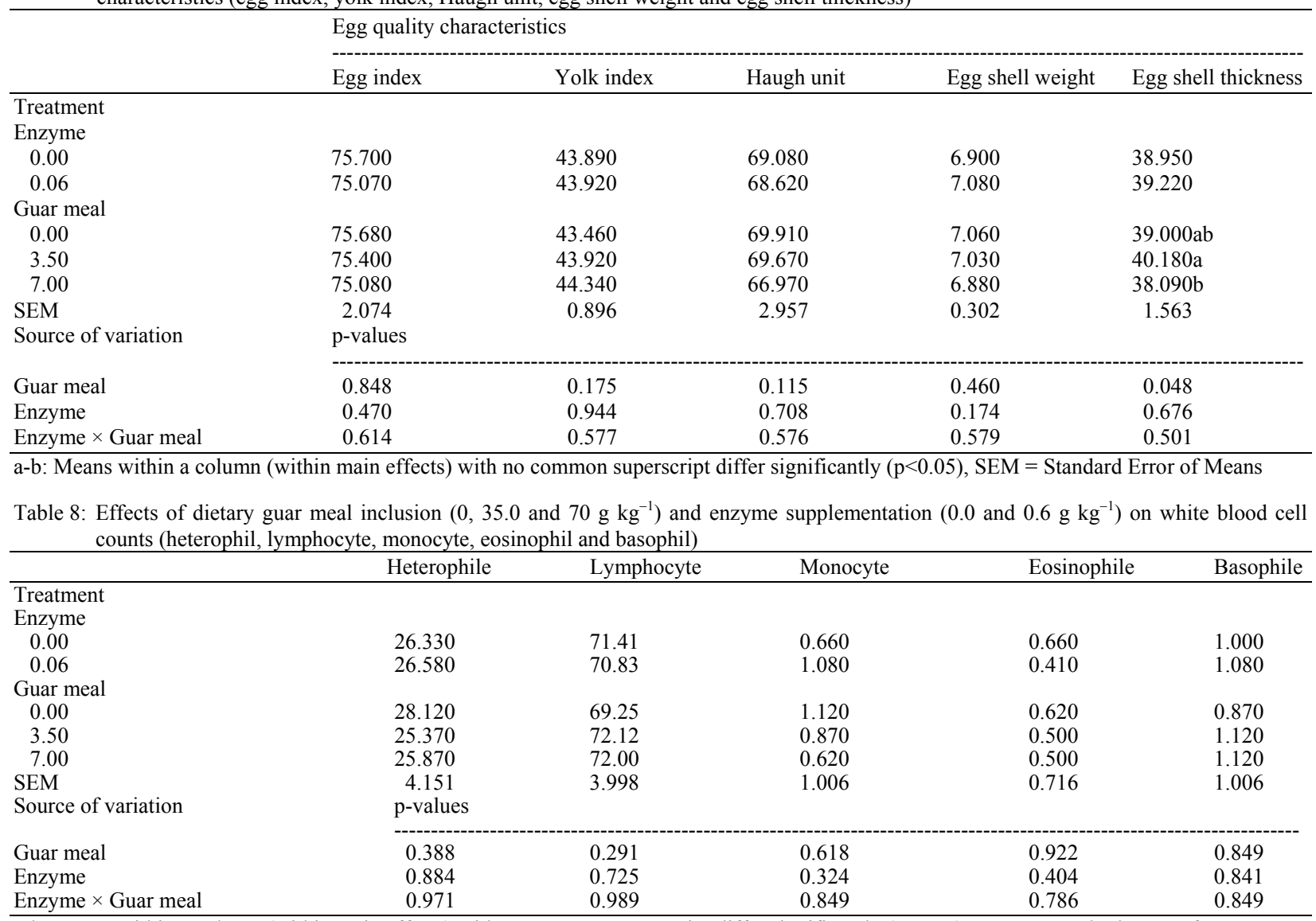

a-b: Means within a column (within main effects) with no common superscript differ significantly ( $<0.05$ ), SEM = Standard Error of Means 


\section{DISCUSSION}

Some studies reported that there was no negative impact on productive performance after adding GM without enzyme to diets at concentrations up to $2.5 \%$ in broiler chicks (Lee et al., 2003a; 2003b) or 5\% in laying hen diets (Gutierrez et al., 2007). Different indigestible polysaccharides, such as pectin, gum Arabic, gum agar, locust bean gum and guar gum, increase intestinal viscosity, which decreases growth and increases feed conversion. Degradation of these gums could allow for by-product meals to be used in poultry diets to decrease the cost of feeding. Generally, viscosity increased with each treatment as digesta traveled through the small intestine from duodenum to jejunum to ileum. Lee et al. (2005) reported that GM can be used at up to $5 \%$ with $\beta$-mannanase enzyme in broilers. Jackson et al. (2004) reported that Hemicell improved weight gain and FCR of broilers.

Hydrolyzing the gum and decreasing digesta viscosity should increase starch digestibility in the small intestine, thereby leading to improved growth and feed efficiency. It has been reported that laying hens induced to molt by GM feeding exhibit improved resistance to Salmonella Enteritidis colonization when compared with hens molted by complete feed withdrawal. Furthermore, supplementation of $\beta$ mannanase (Hemicell) to diets containing high levels of GM appears to enhance resistance to Salmonella Enteritidis colonization in molted laying hens (Gutierrez et al., 2008).

In the study by Lee et al. (2003a), the hull fraction of gaur increased intestinal viscosity at all inclusion levels fed $(0,2.5,5.0,7.5$ and $10.0 \%)$. In their investigation, although FCR was not affected until the inclusion rate exceeded 5.0\%; whereas, the germ fraction significantly increased intestinal viscosity at 7.5 and $10 \%$ inclusion rates. Lee et al. (2005) reported that Hemicell significantly reduced the FCR of guar germ diets to a level comparable with the positive and negative control diets. Daskiran et al. (2004) added Hemicell at $0.5,1$ and $1.5 \%$ in a corn-soybased starter diet containing 1\% GM and found that Hemicell improved FCR at all inclusion levels.

The significant effect of Hemicell on immunity of broilers may be explained by the findings of Wu et al. (2005) who reported that substrate of Hemicell entering the intestinal tract resulted in a reduction of the $\beta$-mannan content associated with a reduction of innate immune stimulation. An important mode of action is a reduction in innate immune stimulation associated with a reduction in the $\beta$-mannan content of substrate entering the intestinal tract. $\beta$-mannans crossing the intestinal mucosa are potent stimulators of the innate immune system, resulting in increased proliferation of macrophages and monocytes and resultant cytokine production.

\section{CONCLUSION}

From the results of the present investigation it can be concluded that including GM in laying hens more than $3 \%$ may cause decrease productive performance. In addition, supplementing corn-soybean or cornsoybean-guar meal diets by $\beta$-mannanase would have beneficial effects on performance of hens especially in terms of feed conversation ratio and egg production.

\section{REFERENCES}

Daskiran, M., R.G. Teeter, D.W. Fodge and H.Y. Hsiao, 2004. An evaluation of endo- $\beta$-D-mannanase (Hemicell) effects on broiler performance and energy use in diets varying in $\beta$-mannan content. Poult. Sci., 83: 662-668. PMID: 15109064.

Fanooci, M. and M. Torki, 2010. Effects of qualitative dietary restriction on performance, carcass characteristics, white blood cell count and humoral immune response of broiler chicks. Global Vet., 4: 277-282.

Ghasemi, R., M. Zarei and M. Torki, 2010. Adding dedicinal herbs including garlic (Allium sativum) and thyme (Thymus vulgaris) to diet of laying hens and evaluating productive performance and egg quality characteristics. Am. J. Anim. Vet. Sci., 5: 151-154. DOI: 10.3844/ajavsp.2010.151.154

Gutierrez, O., C. Zhang, D.J. Caldwell, J.B. Carey and A.L. Cartwright et al., 2008. Guar meal diets as an aternative approach to inducing molt and improving Salmonella enteritidis resistance in latephase laying hens. Poult. Sci., 87: 536-540. DOI:10.3382/ps.2007-00337.

Gutierrez, O., C. Zhang, A.L. Cartwright, J.B. Carey and C.A. Bailey, 2007. Use of guar by product in high production laying hen diets. Poult. Sci., 86: 1115-1120. PMID: 17495081.

Jackson, M.E., K. Geronian, A. Knox, J. McNab and E. McCartney, 2004. A dose-response study with the feed enzyme $\beta$-mannanase in broilers provided with corn-soybean meal based diets in the absence of antibiotic. Poult. Sci., 83: 1992-1996. PMID: 15615012

Lee, J.T., C.A. Bailey and A.L. Cartwright, 2003a. Guar meal germ and hull fractions differently affect growth performance and intestinal viscosity of broiler chickens. Poult. Sci., 82: 1589-1595. PMID: 14601737 
Lee, J.T., C.A. Bailey and A.L. Cartwright, 2003b. $\beta$ mannanase ameliorates viscosity-associated depression of growth in broiler chickens fed guar germ and hull fractions. Poult. Sci., 82: 1925-1931. PMID: 14717550

Lee, J.T., S. Connor-Appleton, C.A. Bailey, A.L. Cartwright, 2005. Effects of guar meal by-product with and without beta-mannanase Hemicell1 on broiler performance. Poult. Sci., 84: 1261-1267. PMID: 16156210

Mahdavi, R. and M. Torki. 2009. Study on usage period of dietary protected butyric acid on performance, carcass characteristics, serum metabolite levels and humoral immune response of broiler chickens. J. Anim. Vet. Adv., 8: 1702-1709. DOI: 10.3923/javaa.2009.1702.1709

Maroufyan, E., A. Kasim, S.R. Hashemi, T.C. Loh and M.H. Bejo, 2010. Change in growth performance and liver function enzymes of broiler chickens challenged with infectious bursal disease virus to dietary supplementation of methionine and threonine. Am. J. Anim. Vet. Sci., 5: 20-26. DOI: 10.3844/ajavsp.2010.20.26
Najafi, P. and M. Torki, 2010. Performance, blood metabolite and immunocompetence of broiler chicks fed diets included essential oils of medicinal plants. J. Anim. Vet. Adv., 9: 1164-1168. DOI: 10.3923/javaa.2010.1164.1168

Wu, G., M.M. Bryant, R.A. Voitle and D.A. S. Roland, 2005. Effects of $\beta$-mannanase in corn-soy diets on commercial leghorns in second-cycle hens. Poult. Sci., 84: 894- 897. PMID: 15971526

Zangiabadi, H.R. and M. Torki, 2010. The effect of a $\beta$ mannanase-based enzyme on growth performance and humoral immune response of broiler chickens fed diets containing graded levels of whole dates. Trop. Anim. Health Prod., 42: 1209-1217. DOI: 10.1007/s11250-010-9550-1

Zangiabadi, H.R. and M. Torki, 2009. Effects of enzyme supplementation of date-induced diets on performance of laying hens. Proceeding of the 2nd Mediterranean Summit of WPSA, Oct. 4-7, Antalya, Turkey, pp: 1-1. 